\title{
Inside Surface Temperature Evaluation of Hollow Blocks Wall by Reduced Linear State Model
}

\author{
Ran Liu ${ }^{\mathrm{a}}$, Yan Gao ${ }^{\mathrm{b}}$, Jing Zhou ${ }^{\mathrm{c}}$ \\ Beijing Key Laboratory of Heating, Gas Supply, Ventilating and Air Conditioning Engineering, Beijing \\ University of Civil Engineering and Architecture, Beijing 100044, China \\ aliuransky@163.com, b'gaoyan@bucea.edu.cn, czhoujing0439@163.com
}

Keywords: Model reduction; hollow blocks; numerical analysis; experimental data.

\begin{abstract}
In this paper, a reduced state space model, based on linear model order reduction methods, is proposed. By this model, hollow block wall can modelling in multi-dimension, dynamic thermal properties can be described clearly, and the variation of internal surface temperature can be simulated accurately and efficiently with external excitation on external wall such as temperature, solar radiation.
\end{abstract}

\section{Introduction}

With the concerns for energy and environment for durable development, more and more buildings envelope are constructed by hollow block for its better energy efficiency and its less lands resources consumption. This kind of envelope has been widespread applied in many countries. And many new innovation are developed to adapt hollow block to the new regulations by optimizing its thermal behavior and others properties [1, 2]. To this type envelope, a great member of theory, numerical, and experimental researches have been represented over this decades. On the aspect of hollow block thermal property, thermal and mass transfer phenomena have been analyzed, and numerous results have been validated from many bibliographic study. In diversity of thermal parameters, U-value is the most important one. Different from mutli-layers wall envelope, U-value of hollow block envelope cannot be easily got with 1-dimensional thermal transfer model owing to its 3-dimensional heat transfer between cavity and block rib, that is to say its heat bridge disturbance.

In National Thermal Code for Civil Building of China, special items about internal surface temperature evaluation have been included for the view of thermal comfortable, and evaluation method has been given. The evaluation attaches important not only static properties, but more dynamic properties of wall. But until now the evaluation method have not indicated very fitful ways for hollow block walls, which have more multi-dimension heat transfer properties than multi-layer wall.

In general, this value is determined by two different procedure, the procedure based on the hot box measurement following either EN ISO 8990 [3] or EN 1934 [4] and second one by two- and three-dimensional numerical calculations according to EN 1745 [5], in Europe. As well known, tabulated U-values for different basic material and perforation pattern are one common means adopted in many country building thermal regulations, such as "Réglementation Thermique 2000" [6] in France. In this way, it is convenient for engineers to determine heat loss of building and as well evaluate energy efficiency of envelope. However, U-value is only a heat loss indication for envelope under static state condition. With the further demands for envelope heat loss evaluate at dynamic state condition, we need more accurately dynamic results of hollow block envelope. But how to deal with the problem for envelope in hollow block is not solved by a simple and acceptable way. And it is probably fair to say that the most general building dynamic thermal analysis programs simplify the thermal conduction problem by assuming one-dimensional (1D) heat flow through walls (DOE-2[5], TRNSYS[6], CODYBA 6.0 [6]). So, it is impossible to accurately obtain dynamic properties of hollow block. Indeed, hollow block envelope does not been included in these programs. Therefore, we need a reasonable model which satisfies for evaluating hollow block envelope. In this paper, we 
propose one simple and practical model by numerical analysis which makes it is possible to accurately and efficiently model dynamic properties of hollow block envelope.

\section{Linear state space model reduction}

State space model. It is not very difficult to set up the differential algebraic equations for a conduction heat transfer problem. For a heat conduction problem, a spatial discretisation of the studied domain $\mathrm{S}$ in $\mathrm{n}$ finite volumes allows us to write a matrix representation of the problem. The centre of each finite volume is the node i which temperature Ti represents the mean temperature of the volume. Then energy balance equation of each node i could be set up according to "Kirckoff law", and the equation is written in the following matrix state-space form:

$$
\dot{T(t)}=C^{-1} \Lambda T(t)+C^{-1} \Pi U(t)
$$

Where:

- $\boldsymbol{T}(\boldsymbol{t})$ is the state vector (dimension: $n, 1)$ : approximation of temperature field, representing the temperature $\mathrm{Ti}$ of each node $\mathrm{i}$ of the spatial discretization.

- $\boldsymbol{C}$ is the square capacitance matrix (dimension: $n, n$ ), diagonal definite positive and Cii elements are the calorific capacities of each control volume.

- $\Lambda$ is a square matrix (dimension: $n, n$ ), which translates the heat exchange between the different control volumes of thermal system $\mathrm{S}$. It is a symmetric matrix because of the reciprocity of the heat transfers.

- $\boldsymbol{U}(\boldsymbol{t})$ is a vector (dimension: $p, 1$ ), which regroups the p solicitations acting on boundary of the studied domain (heat flows, surface or ambiance temperatures).

- $\Pi$ is a rectangular matrix (dimension: $n, p$ ), which translates the thermal relations between the $\mathrm{S}$ domain and its environment. It represents modes of action of solicitations among others on the system.

In general, we are interested in the evolution of some outputs or measures like "the temperature at a particular point", "the flow leaving one domain" etc. All these valuables regroup in one vector Y, which can be expressed under the linear combinations with constant coefficient of temperatures on nodes and inputs. We have:

$$
Y(t)=J T(t)+K U(t)
$$

Where:

$-\boldsymbol{Y}(\boldsymbol{t})$ is the output vector (dimension $q$ : number of observed outputs).

$-\boldsymbol{J}$ is the rectangular observation matrix (dimension: $q, n$ ).

$-\boldsymbol{K}$ is a matrix of direct transmission (dimension: $q, p$ ) which translates the instantaneous actions of solicitations on outputs.

In this way, the dynamic behaviour of a thermal system can be clearly described by the set of matrixes $\mathbf{C}, \boldsymbol{\Lambda}, \boldsymbol{\Pi}, \mathbf{J}, \mathbf{K}$.

However, we know, to our hollow block thermal system modelling in multi-dimensional, system matrixes order are very high because of its numerous differential equations. Moreover, the dynamic simulation process for one multi-dimensional thermal system will be often computationally intensive if we directly apply this high-order matrix model.

Thus, it is necessary to find a simple, low-order model to replace this complete model so that it will benefit to simulation, calculation and computer implantation.

Along with the associated additional computational burdens from high-order model, the simulation and computer implementation may become impossible. The need for low-order model is thus clear. The objective of model size reduction methods is to replace the original complete state model including $\mathrm{n}$ ordinary differential equations( $\mathrm{n}$ is commonly referred to as complete model order) by a significantly smaller one(reduced model of order $m<<n$ ) without sacrificing vital characteristics of the physical system. Model reduction has been the subject of numerous research endeavours and a great member of methods have already been represented over the last 30 years. On that, spectral methods are widely used in buildings thermal simulation domain [7]. Spectral methods represent the 
solution of problems as a linear combination of the Eigen functions of a particular basis. A low-order model is obtained by truncation of the previous state-space coordinate's change. In this paper, balancing realization methods (method of Moore) [8].

Balancing realization method (Moore). Moore method is constructed from the concept of balancing realization technique. This technique is based on the central notions of controllability and observability. The controllability Grampian allows to measure the sensitivity of the state variables to the forcing signals, and the observability Grampian brings a way for measuring the sensitivity of the model outputs to the state variables. In addition, controllability gramian and observability gramian can be obtained from system matrixes. Consequently, reduction of the state variables showing either a weak degree of controllability or a small degree of observability is a possible way for model size reduction. The controllable and observable state elements are defined by their controllability gramian WC and observability gramian WO, obtained by the following Lyapunov equations:

$$
\left\{\begin{array}{c}
\left(C^{-1} \Lambda\right) W_{C}+W_{C}\left(C^{-1} \Lambda\right)^{T}=-\left(C^{-1} \Pi\right)\left(C^{-1} \Pi\right)^{T} \\
\left(C^{-1} \Lambda\right)^{T} W_{O}+W_{O}\left(C^{-1} \Lambda\right)=-J^{T} J
\end{array}\right.
$$

However, the contrill ability and the observability gramians are not system invariant, they strongly depend on the state-space coordinates selected for system behaviour representation. Furthermore, some conflicts among state variables can appear for a state variable showing a weak degree of controllability can show, at the same time, a strong degree of observability, and vice versa. To avoid such difficulties, the initial system is put through a particular similar transformation in order to balance the degrees of observability and controllability. Each one of the state variables in the resulting equivalent model has the same degree of controllability and observability. In other words, the idea for balancing technique is to find a realization to the reduction model, which corresponding observability and controllability gramians are equal and diagonal: $\mathrm{Wc}_{\mathrm{c}}=\mathrm{Wo}_{\mathrm{o}}=\Sigma=\operatorname{diag}(\omega 1, \omega 2, \ldots ., \omega n)$, where $\omega 1 \geq \omega 2 \geq \ldots \geq \omega n>0$ are the so-called Hankel singular[10] values and are the square roots of the eigenvalues of the product WcWo. In a balancing model, the state components corresponding to small Hankel singular values require much energy to be reached, while at the same time producing little energy on the output. Therefore, if there is a $s \in\{1, \ldots, n-1\}$ for which $\omega s>>\omega s+1$, the modal elements corresponding to $\omega \mathrm{m}, \mathrm{m}=\mathrm{s}+1, \ldots, \mathrm{n}$ can be truncated from the system description, preserving only those features of the dynamics that are most relevant to observable and controllable modals. Model size reduction is then performed by simple elimination of the modal parts associated to the state variables showing weak observability and controllability degrees.

After the calculation of grammiens of observability and controllability and balancing transformations, expression of system in this base is in follow equation:

$$
\begin{aligned}
& \left\{\begin{array}{l}
\dot{X}_{1} \\
\dot{X}_{2}
\end{array}\right\}=\left[\begin{array}{ll}
\Omega_{11} & \Omega_{12} \\
\Omega_{21} & \Omega_{22}
\end{array}\right]\left\{\begin{array}{l}
X_{1} \\
X_{2}
\end{array}\right\}+\left[\begin{array}{l}
B_{1} \\
B_{2}
\end{array}\right] U \\
& Y=\left[\begin{array}{ll}
H_{1} & H_{2}
\end{array}\right]\left\{\begin{array}{l}
X_{1} \\
X_{2}
\end{array}\right\}+D U
\end{aligned}
$$

Conserving m modals which have the most observable and controllable, the reduction model is expressed by Eq. 5 :

$$
\left\{\begin{array}{l}
X_{M O}=A_{M O} X_{M O}+B_{M O} U \\
Y=H_{M O} X_{M O}+D_{M O} U
\end{array}\right.
$$

$\boldsymbol{A}_{\text {мо }}, \boldsymbol{B}_{\text {мо }}, \boldsymbol{H}_{\text {мо }}, \boldsymbol{D}_{\text {мо }}$ are reduced system model matrixes, where

$$
\begin{aligned}
& A_{M O}=\Omega_{11}-\Omega_{12} \cdot \Omega_{22}^{-1} \cdot \Omega_{21} ; B_{M O}=B_{1}-\Omega_{12} \cdot \Omega_{22}^{-1} \cdot B_{2} ; \\
& H_{M O}=H_{1}-H_{2} \cdot \Omega_{22}^{-1} \cdot \Omega_{21} ; D=D-H_{2} \cdot \Omega_{22}^{-1} B_{2} .
\end{aligned}
$$




\section{Numerical simulation}

One usual hollow blocks was used in this paper, two ranges cavity block. Considering rectangular mesh discretization, the arc part and cone-shaped part are simplified as rectangle just like shown in Fig.1
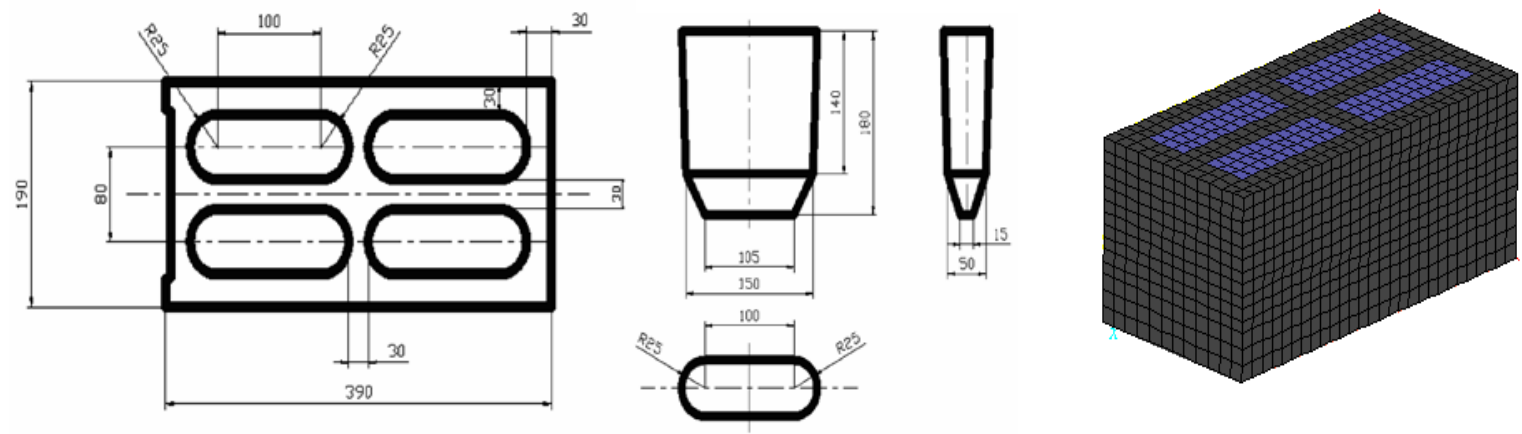

Fig. 1 Plan view, cross section and discretization of hollow block

Before numerical simulation, air layer equivalent thermal conductivity is determined by numerical simulation [10] Consequently, the air layer equivalent thermal conductivity $\left(\lambda_{e q}\right)$ of hollow blocks, is taken as $0.28 \mathrm{~W} / \mathrm{m}^{2} \mathrm{~K}$ within our numerical model.

According to national thermal code for civil building [11], inside surface temperature of east, west wall and roof should satisfy Eq.6, for thermal comfort consideration in summer.

$\theta_{i \cdot \max } \leq t_{e \cdot \max }$

$\theta_{\boldsymbol{i} \cdot \max }:$ max temperature of inside surface; $t_{e \cdot \max }$ : max outside air temperature for design

The mathematic method for validation is given by this design criterion.

For ordinary multi-layer wall, heat transfer in one-dimensional, the method given by the code is helpful and available. Unfortunately, it can't deal with the wall with thermal bridge characterized multi-dimensional heat transfer, just like hollow block. Consequently, model reduced method is used for validation of inside wall surface temperature.

The outside boundary condition of hollow block is given by Eq.7

$t_{s a}=t_{e}+\alpha I / h_{e}$

$t_{s a}$ : solar-air temperature of outside; $t_{e}$ : outside air temperature; $I$ : is total incident solar radiation on outside surface; $\alpha$ : absorptance of the outside of surface for solar radiation; $h_{e}$ : outside heat exchange coefficient.

The boundary condition for simulation is given in Table 1.

Table 1 Boundary condition for simulation

\begin{tabular}{ccccc}
\hline $\begin{array}{c}\text { Average outside } \\
\text { air temperature } t_{\boldsymbol{e}}\end{array}$ & $\begin{array}{c}\text { Outside surface } \\
\text { convection } \\
\text { coefficient } h_{\boldsymbol{e}}\end{array}$ & $\begin{array}{c}\text { Average inside } \\
\text { air temperature } t_{\boldsymbol{i}}\end{array}$ & $\begin{array}{c}\text { Inside surface } \\
\text { convection } \\
\text { coefficient } h_{\boldsymbol{i}}\end{array}$ & $\begin{array}{c}\text { Solar } \\
\text { radiation on } \\
\text { surface } I\end{array}$ \\
\hline $31.1 \mathrm{C}$ & 19 & $32.6 \mathrm{C}$ & 8.7 & $\begin{array}{c}\text { Shown in } \\
\text { Fig.2 }\end{array}$ \\
\hline
\end{tabular}

Note: the value used is according to the code given for Guangzhou located in South of China.

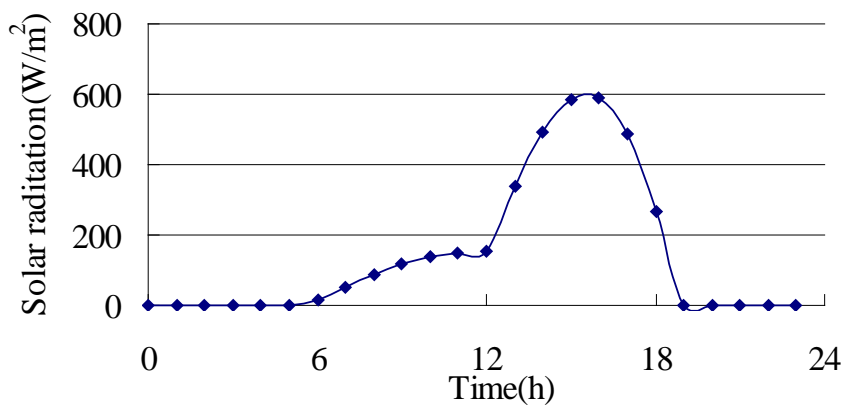

Fig.2 Solar radiation on surface 
The thermal properties for hollow block is given in Table 2,

Table 2 Thermal properties of hollow block

\begin{tabular}{cccc}
\hline & $\lambda(\mathrm{W} / \mathrm{m} \cdot \mathrm{K})$ & $\rho(\mathrm{kg} / \mathrm{m} 3)$ & $\mathrm{c}(\mathrm{J} / \mathrm{kg} \cdot \mathrm{K})$ \\
\hline $\begin{array}{c}\text { hollow block (concrete } \\
\text { part) }\end{array}$ & 1.75 & 2000 & 1000 \\
$\begin{array}{c}\text { hollow block (air layer } \\
\text { part) }\end{array}$ & 0.278 & 1.15 & 1005 \\
\hline
\end{tabular}

Numerical analysis was carried out by our state-space complete model Eq.1, 2 and Moore reduced model Eq. 5 for hollow block thermal system. Reduced model of our case has only $\mathbf{5}$ modals, compared with complete model having 1601 modals. On this aspect, this reduced model has satisfying high efficiency. Then, we compare simulation results from complete and reduced model. In Fig.3, two curves coming from different model are very close, relatively errors is less $0.1 \%$. From this point, reduced model is very efficient and the calculation time is far less than complete model.

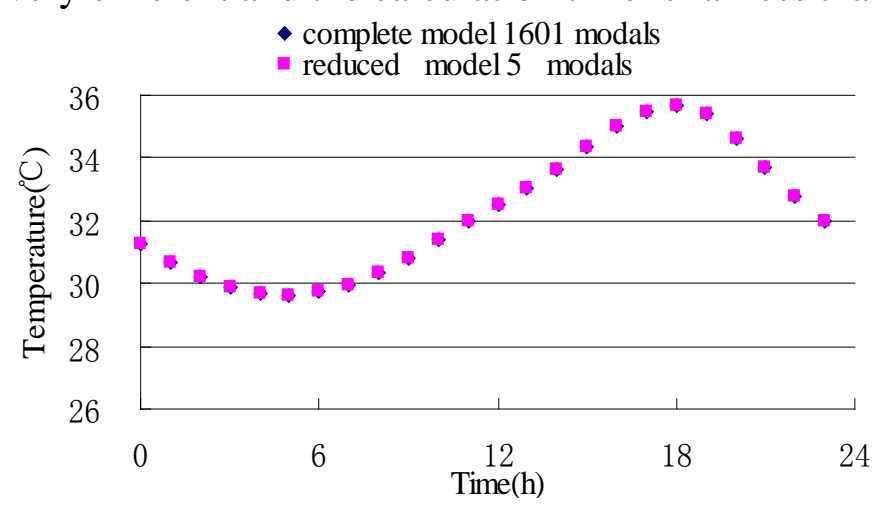

Fig.3 Temperature from different model

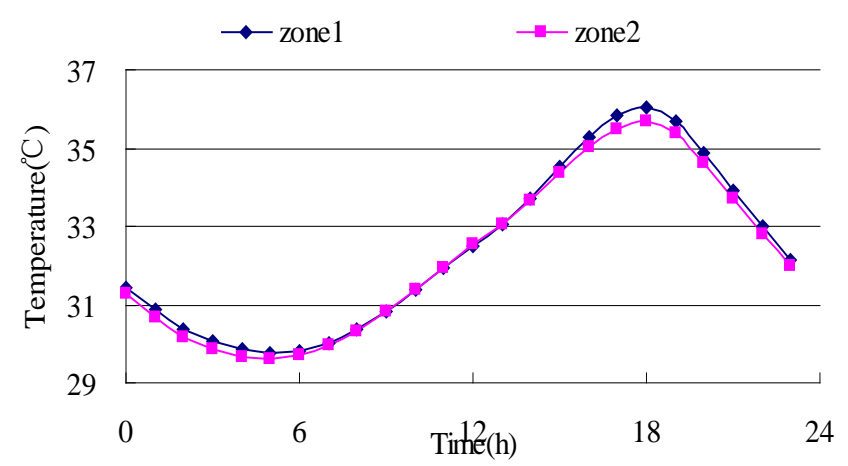

Fig. 4 Tempetature at different zones

Temperature distribution at hollow block inside surface is not uniform like multi-layer wall, surface temperature at different position may be uneven. Figure 4, gives different zone of block inside surface. Temperature at the rib position (zone1) is lightly higher than the position against air layer (zone2) because of the thermal bridge, thermal resistance at zone1, rib position, is less than zone2. At the morning time, the temperature is nearly same between two zones. The time (zone1 temperature higher than zone2) begins from $15 \mathrm{~h}$ afternoon, when outside surface has intensive solar radiation and inside surface has max temperature at 18 owing the thermal inertia of concrete. Furthermore, the temperature difference of the two zone is less than $0.5 \mathrm{C}$. This slight difference shows the thermal bridge effect of this hollow block is not very important for thermal comfort at summer.

In summary, Moore reduced model is accurate and efficient enough to be used for characterizing building envelope thermal transfer performance concerned by us. We can use model reduction to deal with all kinds of hollow block, and obtain their reduced model in the form of very low order state 
space matrixes. These matrixes can be easily implanted in program as a model library. So, this library make it possible for us to analyse thermal performance of hollow block envelope in simple and efficient way.

\section{Summary}

Three-dimensional heat transfer character in hollow block makes it impossible to use traditional multi-layers 1-dimensional model to simulate heat loss through this type envelope under dynamic state condition. However, complete 3-dimensional model which directly come from thermal system differential equations has a very high matrix order. This high-order model restrain its application for its complexity. In this paper, we propose the possibility to use model size reduction techniques for constructing reduced model which is fit for implantation in computer. For that, thermal simulation calculation for hollow block envelope will be simple just like looking for a tabulate value. Moreover, efficiency and accuracy of reduced model has satisfying results.

\section{Nomenclature}

$\begin{array}{llll}\mathrm{A}, \mathrm{B}, \mathrm{H}, \mathrm{D} & \text { reduced model matrixes } & \text { Subscripts } & \\ \mathrm{C}, \Lambda, \Pi, \mathrm{J}, \mathrm{K} & \text { complete model matrixes } & \mathrm{a} & \text { air } \\ \mathrm{N}_{\mathrm{u}} & \text { Nusselt number } & \text { eq } & \text { equivalence } \\ \mathrm{Ra} & \text { Rayleigh number } & \text { conv } & \text { convection } \\ \lambda & \text { thermal conductivity }(\mathrm{W} / \mathrm{mK}) & \mathrm{rad} & \text { radiation } \\ \sigma & \text { Stefan Bolzmann constant 5.67E-8W/m}{ }^{2} \mathrm{~K}^{4} & \mathrm{MO} & \text { Moore method } \\ \varepsilon & \text { emissivity emittance /absorptance coefficient } & & \end{array}$

\section{References}

[1] A. G. Loudon, The thermal properties of lightweight concretes, International Journal of Cement Composites and Lightweight Concrete 1 (2) (1979) 71-85.

[2] Y. Dingyi, S. Wei, L. Zhiyong, Z. Keren, Research on improving the heat insulation and preservation properties of small-size concrete hollow blocks, Cement and Concrete Research 33 (2003) 1357-1361.

[3] EN ISO 8990, Thermal insulation: determination of steady-state thermal transmission properties, Calibrated and guarded hot box.

[4] EN 1934, Thermal performance of building: determination of thermal resistance by hot box method using heat flow meter, Masonry.

[5] EN 1745, Masonry and products: methods for determining design thermal values.

[6] Réglementation Thermique 2000, 2000, CSTB (Centre Scientifique et Technique du Bâtiment), Paris, France.

[7] D. Petit. Réduction de modèles de connaissance et identification de modèles d'ordre réduit, Ph.D. Dissertation, 1991, Université de Provence (Aix-Marseille I), France.

[8] B. C. Moore, Principal component analysis in linear systems: controllability, observability, and model reduction, IEEE Trans. Automatic Control 26 (1) (1981) 17-32.

[9] EN ISO 6946, Building components and building elements: thermal resistance and thermal transmittance, Calculation method.

[10] Y. Gao, J.J. Roux, C. Teodosiu, L.H. Zhao. Reduced linear state model of hollow blocks walls validation using hot box measurements, Energy and Buildings 36 (2004) 1107-1115 
[11] Thermal design code for civil building, GB 50176-93, 1993, China State Bureau of Quality and Technical Supervision\& Ministry of Construction, Beijing, China. 